PANORAMA ECONÓMICO, Volumen VI, No. 11, julio-diciembre, 2010, pp. 7-28

\title{
VALUACIÓN FINANCIERA DE PROYECTOS DE ENERGÍA NUCLEAR EN ARGENTINA MEDIANTE OPCIONES REALES
}

\author{
Francisco Álvarez Echeverría* \\ Francisco Venegas-Martínez** \\ Pablo López Sarabia***
}

(Recibido: Julio, 2010 / Aprobado: Octubre, 2010)

\begin{abstract}
RESUMEN
El cambio climático y el agotamiento de los combustibles fósiles han llevado a la política energética hacia las prioridades del panorama estratégico mundial. La discusión se concentra en el suministro de combustibles para economías tanto desarrolladas como en vías de desarrollo, considerando que las tecnologías renovables son costosas con respecto a las que utilizan combustibles fósiles. No obstante, el empleo de la energía nuclear resulta financieramente viable en relación a otras tecnologías energéticas renovables. Esta investigación utiliza opciones reales para valuar proyectos de inversión en energía nuclear para el caso de Argentina, quien es líder en la operación de plantas nucleares en América Latina.

Palabras clave: Energía nuclear, opciones reales, combustibles fósiles y valuación de proyectos

Clasificación JEL: G1, G13, G31, O32

* Profesor de la División de Estudios de Posgrado de la Facultad de Ingeniería, UNAM. Correo electrónico:<franlve@yahoo.com.mx>.

** Profesor-Investigador de la Escuela Superior de Economía, ESE-IPN. Correo electrónico: $<$ fvenegas1111@yahoo.com.mx>.

***Profesor-Investigador del Instituto Tecnológico y de Estudios Superiores de Monterrey, Campus Estado de México, ITESM-CEM. Correo electrónico: <plopezs@itesm.mx>.
\end{abstract}




\begin{abstract}
Climate change and depletion of fossil fuels have led to energy policy to the priorities of the global strategic scenery. The discussion focuses on the supply of fuel for economies, both developed and developing, considering that renewable technologies are expensive compared to using fossil fuels. However, the use of nuclear energy is financially viable in relation to other renewable energy technologies. This research uses real options to value investment projects in nuclear energy in the Argentinean case, who is leading the operation of nuclear plants in Latin America.

Keywords: Nuclear energy, real options, fossil fuels and valuation of projects JEL Classification: G1, G13, G31, O32
\end{abstract}

\title{
1. INTRODUCCIÓN
}

La mayoría de las economías desarrolladas o en vías de desarrollo siguen siendo altamente dependientes de las energías fósiles no renovables, particularmente del petróleo y del gas natural, situación que explica el desequilibrio existente en la matriz energética de dichos países. En contraste con esta situación, los Estados Unidos de Norte América generan, en la actualidad, el 30\% de su energía con fuente limpias y renovables, entre las que destaca la energía nuclear, la cual aunada al desarrollado de nuevas tecnologías la hacen económicamente más eficiente y condescendiente con el medio ambiente. La implementación de un proyecto núcleo-energético implica, sin lugar a dudas, tomar en cuenta todos los sectores de la economía y actores dentro de una sociedad, por lo que es de vital importancia contar con técnicas y herramientas que permitan una valuación financiera apropiada de este tipo de tecnologías energéticas.

Por otro lado, la volatilidad experimentada por los precios de los hidrocarburos demuestran la vulnerabilidad de la mayoría de las economías, tanto desarrolladas como en vías de desarrollo, en lo referente a la implementación de políticas económicas de mitigación de los efectos inflacionarios (adversos) producidos por 
VALUACIÓN FINANCIERA DE PROYECTOS DE ENERGÍA NUCLEAR

en Argentina mediante opciones reales

los incrementos de los combustibles fósiles, sobre los cuales se carece de control y que, a su vez, impactan los costos de las empresas y los ingresos familiares. Las lecciones de la crisis del petróleo de 1973, la cual inicia con la suspensión de envíos de petróleo por parte de la Organización de los Países Exportadores de Petróleo (OPEP) a los países que habían apoyado a Israel durante la guerra del Yom Kippur, entre los que se encontraban los Estados Unidos y otros países de Europa Occidental, disparó los precios internacionales del petróleo a casi el doble en unos días, lo cual, sumado a la gran dependencia de combustibles fósiles del mundo industrializado, provocó una espiral inflacionaria a gran escala y una reducción de la actividad económica de los países involucrados.

En la actualidad, el uso de las fuentes de energía tradicionales (petróleo y sus derivados) es preocupante, no sólo porque de ellos depende una gran parte de la economía mundial, sino también por los altos costos que involucra su adquisición, como en el caso de la perforación de pozos en aguas profundas, y por los efectos negativos que han provocado en el medio ambiente a lo largo de las últimas décadas, como lo muestra la reciente debacle ecológica ocasionada por British Petroleum (BP) en el Golfo de México. Razón por la que se debe dar un impulso decisivo a la adopción de nuevas tecnologías energéticas que sean más eficientes, menos contaminantes, pero sobre todo sostenibles en el largo plazo.

Los biocombustibles se han popularizado como un mecanismo para evitar la dependencia económica de combustibles fósiles. Sin embargo, se han producido efectos adversos en el sector alimentario ya que, la producción de combustible a partir de productos agropecuarios pone en riesgo el abastecimiento de productos básicos para el consumo humano, más aún si se consideran los efectos negativos en la erosión de grandes extensiones de tierra dedicadas a la producción de maíz, trigo y caña de azúcar, utilizados para la producción de etanol y biodisel.

En lo referente a la energía nuclear, ésta ha cobrado un nuevo impulso ante el desarrollo tecnológico de una nueva generación de reactores más eficientes y menos agresivos con el medio ambiente y que no generan gases de efecto invernadero. En la actualidad existen, en el mundo, casi 500 reactores nucleares para la generación de electricidad, los cuales producen el 17\% de la electricidad mundial. Asimismo, para 2010 se tenían contempladas 56 nuevas unidades en países como 
China, India, Bulgaria, Japón, Rusia, Corea del Sur, Finlandia y Francia, por mencionar algunos de los más importantes, a lo que debemos sumar las centrales ya planificadas que ascienden a más de 200. En el caso de América Latina, el líder en la generación de electricidad con reactores nucleares es Argentina, con dos reactores en operación y uno más en construcción.

En la actualidad, la energía nuclear es una alternativa viable ante la amenaza del calentamiento global y del cambio climático, esto aunado al incremento de la demanda de electricidad y del precio de los productos petrolíferos. Esta investigación utiliza la técnica de opciones reales para la valuación económica y financiera de un proyecto núcleo-energético para el caso de Argentina, líder en dicha tecnología en América Latina. Así mismo, se llevará a cabo una simulación que permitirá contrastar otras tecnologías energéticas disponibles.

\section{LA ENERGÍA NUCLEAR Y TIPOS DE REACTORES NUCLEARES}

Los costos de generación de energía nuclear dependen en gran medida del tipo de reactor nuclear que se utilice, por ejemplo, en las instalaciones industriales en donde se requieren reactores de potencia, éstos desempeñan el mismo papel que las calderas en las instalaciones de tipo tradicional. La diferencia consiste en el hecho de que mientras en una caldera el combustible (aceites pesados o carbón) posee un contenido energético específico relativamente bajo y, por lo tanto, debe ser aprovisionado con continuidad (durante la vida de la instalación), en el caso de una caldera nuclear el combustible posee un contenido energético específico tan elevado que una carga completa del mismo garantiza su funcionamiento durante varios años. Por esta razón, el reactor puede considerarse como un gran depósito de combustible.

Es importante destacar que las instalaciones en las que se emplean los reactores nucleares son similares a las de tipo tradicional en muchos aspectos. En general, el fluido que trabaja en el ciclo industrial se obtiene indirectamente a través de intercambiadores de calor a partir del fluido de refrigeración del reactor, y no faltan los casos de utilización directa o mixta del propio fluido de refrigeración 


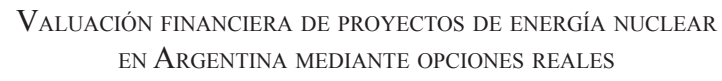

(López Álvarez, 2006). De acuerdo a lo anterior podemos clasificar los tipos de reactores de la siguiente manera: 1) reactores de la primera generación, 2) reactores de segunda generación, 3) reactores de tercera generación y 4) reactores de cuarta generación.

\subsection{REACTORES DE PRIMERA GENERACIÓN}

En la segunda mitad del siglo XX se comenzaron a construir los primeros prototipos y diseños de reactores nucleares, etapa ahora llamada Generación I. A partir de este momento se puede decir que la evolución de los reactores nucleares ha pasado por diferentes fases, denominadas con el término de generaciones. Por lo general, aunque la complejidad del reactor pueda ser elevada, al final se trata de una fuente de calor como otra cualquiera, y los ciclos utilizados en este tipo de centrales son similares a los de otras centrales. Los más utilizados comercialmente son ciclos de vapor de agua, aunque en la actualidad se está experimentando más con ciclos de gases como el Helio o el $\mathrm{CO}_{2}$.

\subsection{REACTORES DE LA GENERACIÓN II}

Los primeros reactores comerciales (Generación II) empezaron a operar en 1965. Aunque todavía existen muchas centrales nucleares que actualmente están en funcionamiento y que disponen de reactores de este tipo: de agua pesada (CANDU), de agua presurizada (PWR), de ebullición de agua (BWR), de sodio grafito, de gas (HGTR) y de cría (LMFBR).

El Reactor Refrigerado por Gas (HGTR) se caracteriza por que el moderador es de grafito y el refrigerante de bióxido de carbono gaseoso, el cual está contenido en un recipiente a presión de hormigón, que también actúa como escudo. La turbina generadora es impulsada por vapor producido en una caldera. Durante el proceso no se deben acumular grandes cantidades de calor.

El Reactor de Agua en Ebullición (BWR) utiliza agua natural purificada como moderador y refrigerante. Como combustible dispone de Uranio-238 enriquecido con Uranio-235, el cual, como se sabe, facilita la generación de fisiones nuclea- 
res. El calor generado por la reacciones en cadena se utiliza para hacer hervir el agua. El vapor producido se introduce en una turbina que acciona un generador eléctrico. El vapor que sale de la turbina pasa por un condensador donde es transformado nuevamente en agua líquida, posteriormente vuelve al reactor al ser impulsada por una bomba adecuada.

El Reactor de Agua a Presión (PWR). En este caso, el refrigerante es agua a gran presión. El moderador puede ser agua o bien grafito. Su combustible también es Uranio-238 enriquecido con Uranio-235. El reactor se basa en el principio de que el agua sometida a grandes presiones puede evaporarse sin llegar al punto de ebullición, es decir, a temperaturas mayores de $100{ }^{\circ} \mathrm{C}$. El vapor se produce a unos $600{ }^{\circ} \mathrm{C}$, el cual pasa a un intercambiador de calor donde es enfriado y condensado para volver en forma líquida al reactor. El agua pasa a un circuito secundario y como producto del calor se genera vapor que se introduce en una turbina que acciona un generador eléctrico.

El Reactor de Cría (LMFBR), se denomina de esta manera por que produce por medio de neutrones procedentes en exceso de las fisiones en cadena, de núcleos no fisionables, isótopos fisionables del mismo o de distinto material. Produce combustible al mismo tiempo que lo quema, el cual es reutilizado en futuras recargas.

\subsection{REACTORES DE LA GENERACIÓN III}

Se ha denominado con este término a los reactores de Generación II que son reactores evolutivos refrigerados por agua ligera. Esta fase se desarrolla durante la década de los noventa y los reactores son más eficientes y avanzados basándose en tecnologías existentes. En estos reactores se empiezan a diseñar los sistemas de seguridad avanzados que caracterizan el futuro de las centrales nucleares y que dieron paso a una filosofía llamada "seguridad a ultranza" o "Defense In Depth". Entre ellos destacan los sistemas de seguridad pasivos que conllevan características de seguridad basadas en la convección y la gravedad, lo que permite reducir la dependencia de las funciones de seguridad de los sistemas y componentes activos tales como las bombas y válvulas. Esta generación se basa principalmente en 
VALUACIÓN FINANCIERA DE PROYECTOS DE ENERGÍA NUCLEAR

en Argentina mediante opciones Reales

reactores refrigerados por agua, aunque aquí mismo se puede clasificar el Reactor Modular de Lecho de Bolas (Pebble Bed Modular Reactor PBMR) como una evolución del Reactor de Alta Temperatura (HTR), con lo que quedaría enmarcado dentro del contexto de la llamada Generación III+, encontrándose a medio camino entre las Generaciones III y IV.

\subsection{REACTORES DE LA GENERACIÓN IV}

En la Generación IV se engloba toda una serie de proyectos, programas e iniciativas para el desarrollo y prueba de varios sistemas nucleares, utilizables comercialmente hacia 2030 y que ofrecen ventajas en los campos de sostenibilidad, economía, seguridad y fiabilidad. Además de la generación de energía eléctrica directa en centrales, es aplicable a otros campos como la producción de hidrógeno o grandes sistemas de transporte. Uno de los retos más ambiciosos de este proyecto es el de sostenibilidad, basado en la disminución o eliminación de los residuos radiactivos mediante el uso de reactores rápidos o técnicas de transmutación. En cuanto a los aspectos económicos, se espera aumentar la vida de estas centrales a 60 años, con lo que se consiguen costos competitivos, y el aumento de los sistemas de seguridad con niveles de riesgos financieros comparables a otros proyectos energéticos. Los principales reactores que se están desarrollando en la actualidad son: SFR, Reactor Rápido Refrigerado por Sodio; LFR, Reactor Rápido Refrigerado por Aleación de Plomo; GFR, Reactor Rápido Refrigerado por Gas; y SCWR, Reactor Súper Crítico Refrigerado por Agua.

\subsection{COSTOS Y VIABILIDAD DE LA ENERGÍA NUCLEAR}

Con respecto a los costos de producción de energía con base en núcleo-eléctricas, Bazán-Perkings (2005) afirma que, en los Estados Unidos las termoeléctricas con base en la energía nuclear y carbón mineral promedian, desde hace varias décadas, costos de generación (operación, mantenimiento y combustible) menores al que utilizan el petróleo y el gas como combustible, de estas cuatro tecnologías (gas, nuclear, carbón y petróleo) la nuclear es la de menor costo. En efecto, al 2001 los 
costos de generación con base en gas eran de unos 5.69 \$US/kWh, las de petróleo de 5.28 \$US/kWh, de carbón 1.79 \$US/kWh y nuclear de $1.76 \$ \mathrm{US} / \mathrm{kWh}$, siendo la energía nuclear la más competitiva, después de las grandes hidroeléctricas, con costo de 1.2 \$US $/ \mathrm{kWh}$. Actualmente, los costos de inversión típicos en plantas nucleoeléctricas nuevas se encuentran entre los 1000 y 1400 \$US/Kw. Dicho valor es aún alto al contrastarlo con los 500-600 \$US/kW que requieren las de ciclo combinado con base en gas. Asimismo, para promover la inversión en plantas núcleo-eléctricas nuevas, el gobierno de los Estados Unidos apoya su financiamiento hasta en un 50\%. Adicionalmente, los costos de generación de las plantas nucleares de reciente instalación han tenido un rápido descenso, de 1996 al 2003 pasaron de los 7.1 \$US/kWh a un $3.3 \$ \mathrm{US} / \mathrm{kWh}$. Se proyecta que hacia el 2010 los reactores de Generaración III+ tendrán costos entre 1.6 a 3.5 \$US/kWh. Entre tanto, hacia el 2015, los de Generación IV entrarán al mercado con costos por debajo de los 3 \$US/kWh. En suma, para 2003, los costos totales nivelados de las plantas núcleo-eléctricas de Generación III estarían entre los 3.2 y 3.7 \$US/kWh. En Argentina estos costos son equiparables a los de las nuevas plantas de ciclo combinado a base de gas, del orden de 2.5 y $3.6 \$ \mathrm{US} / \mathrm{kWh}$. Además, la viabilidad económica de las núcleo-eléctricas se favorece por su mayor factor de planta, alcanzan un $92 \%$, mientras que las de ciclo combinado son de $80 \%$. En estas condiciones, por ejemplo, si ambas tecnologías participaran con costos similares, la de mayor factor de planta sería más viable: la núcleo-eléctrica.

En la República de Argentina la expansión de la capacidad de generación eléctrica instalada requerirá en los próximos años una fuerte inversión para cubrir la creciente demanda del servicio a nivel nacional. Asimismo, los altos costos por el consumo de hidrocarburos propician que la generación de electricidad en plantas nucleares sea un camino viable. De igual manera, la utilización de este tipo de energía alterna reduce la emisión de gases invernadero, permitiendo así una disminución importante en los niveles de contaminantes en la atmósfera y en la consecución de metas en el marco de tratados internacionales dentro de éste ámbito.

Por lo anterior se ha decidido realizar una evaluación económica y financiera entre las fuentes alternas de energía mediante el uso de opciones reales para Ar- 
VALUACIÓN FINANCIERA DE PROYECTOS DE ENERGÍA NUCLEAR

en Argentina mediante opciones REALES

gentina, país líder en energía nuclear en América Latina, que cuenta con información de las diversas tecnologías de manera detallada.

\section{EVALUACIÓN DE PROYECTOS Y LAS OPCIONES REALES}

La sobrevivencia y factibilidad de proyectos de inversión que dependen del desarrollo tecnológico requieren de una proyección efectiva del capital de trabajo, así como de una adecuada planeación que permita contar con proyecciones confiables de los posibles beneficios que se puedan generar. Los métodos tradicionales de evaluación de proyectos resultan ineficientes cuando existe un nivel alto de incertidumbre dentro de los proyectos, ya que dichos métodos sólo pueden ser utilizados para la evaluación de proyectos de corto plazo en mercados con una relativa certidumbre en el largo plazo. En consecuencia, es imprescindible utilizar métodos más generales que permitan cierta flexibilidad en el manejo futuro de las inversiones.

El valor de los proyectos con un grado alto de incertidumbre (sobre todo en proyectos energéticos, debido al costo de la tecnología y al precio de los carburantes, según sea el caso) no consiste simplemente en agregar o descontar flujos de efectivo, sino también en considerar las oportunidades en el futuro, tales como: expansión de la inversión, contracción de la producción, abandono del proyecto, por mencionar algunas, las cuales a final de cuentas, resultan ser meramente opciones (que podrían ejercerse en el futuro) dentro de la estrategia corporativa. Mediante el criterio del valor presente neto (VPN) un proyecto nuevo o una nueva estrategia se acepta o se rechaza si VPN $>0$ ó VPN $<0$, respectivamente, y no existe otra posibilidad. La implementación de proyectos energéticos en la actualidad, requiere de proyecciones confiables de los posibles beneficios, aunque el método VPN resulta ser muy sencillo de emplear en la práctica presenta la desventaja de que algunas inversiones futuras podrían quedar rechazadas o latentes, sin tomar en cuenta su valor estratégico a través del tiempo (MacDougall y Pike, 2003).

Los proyectos energéticos se caracterizan por estar inmersos en horizontes de planeación de largo plazo con un alto grado de incertidumbre, en donde el valor de la flexibilidad administrativa puede ser vital. Dentro de este marco, desde 
hace algún tiempo se han venido utilizando herramientas financieras que permiten establecer cierta flexibilidad en los planes de inversión, mediante el uso de un horizonte mucho más amplio que los métodos actuales. De esta forma, Brealey y Myers (1991) señalan que las opciones reales permiten agregar cierto valor a la empresa a través de la flexibilidad (opcionalidad) en las inversiones futuras. Cuando se utilizan las técnicas de valuación de proyectos como el VPN u otras de las técnicas tradicionales, éstas son incapaces de administrar decisiones futuras en los planes de inversión (Lander y Pinches, 1998). Es por lo anterior, que algunos autores consideran que el tiempo óptimo de la adopción tecnológica puede ser representado mediante un modelo de irreversibilidad en la inversión, en dónde el VPN resulta ser una solución sub-óptima (Doraszelski, 2001).

\subsection{LAS OPCIONES REALES}

La metodología de valuación financiera de proyectos mediante la utilización de opciones reales se ha convertido en una herramienta indispensable para la toma de decisiones en proyectos de inversión o estrategias de negocios cuando existe la flexibilidad u opcionalidad de tomar nuevas decisiones relacionadas con dichos proyectos o estrategias en una fecha futura, como pueden ser: la extensión, contracción, posposición, corrección o abandono de un proyecto. La idea de las opciones reales es incorporar una opción financiera al esquema de valuación de un proyecto de inversión con la siguiente correspondencia entre los parámetros de una opción financiera y los de una opción real. Una empresa, a partir de un tiempo $t$, posee una oportunidad de invertir en un proyecto subyacente hasta el tiempo $T$, lo cual podría verse como una opción para adquirir un cierto valor presente de los flujos esperados, $S_{T}$, a cambio una inversión, $K$, en la fecha de vencimiento. En este caso, $S_{T}$ tiene asociado un factor de incertidumbre, a saber, la volatilidad de los flujos de efectivo del proyecto. Por supuesto que la opción solamente será ejercida cuando $S_{T}>K$, en cuyo caso el inversionista permanece en el proyecto subyacente, de lo contrario sería conveniente abandonarlo. Esto lleva a inferir que, mientras las opciones financieras tratan con activos financieros, las opciones reales tratan con activos reales como pueden ser: unidades de negocio, obras e 
VALUACIÓN FINANCIERA DE PROYECTOS DE ENERGÍA NUCLEAR

en Argentina mediante opciones Reales

infraestructura, nuevas tecnologías o cualquier otro tipo de activos generados a través de proyectos de inversión (Venegas-Martínez, 2006).

Las opciones reales, a diferencia de las opciones financieras, no consideran series históricas de tiempo para estimar la volatilidad sobre el activo subyacente. Además de que en las opciones reales, el valor presente puede ser estocástico. Sin embargo, la incertidumbre en la estimación del valor del subyacente no cambiará el valor de la opción en el presente, ni tampoco la regla de inversión en la maduración de la opción, la cual realizará la inversión cuando la opción se encuentra dentro del flujo de efectivo en el tiempo de maduración; aunque en el caso de tener un valor del subyacente de naturaleza estocástica, la opción podría estar fuera de toda consideración de inversión después de que el producto o el servicio han sido introducidos (Pennings y Lint, 1997). Dentro de las opciones reales podemos encontrar las siguientes: 1) opción real de expansión, 2) opción real de contracción, 3) opción real de cierre temporal, 4) opción real de permanencia, 5) opción real de abandono y 6) opción real de cambio tecnológico.

\subsection{OPCIÓN REAL DE EXPANSIÓN}

La valuación financiera de proyectos de inversión puede considerar diversas clases de opciones reales existentes dentro de la literatura (Lander y Pinches, 1998; Venegas-Martínez, 2006). Es importante destacar que en el caso de valuación con opciones reales, la tasa de descuento es una tasa libre de riesgo de incumplimiento. Una oportunidad corporativa de inversión se puede valuar como una opción (europea o americana) de compra ya que la empresa adquiere la posibilidad de llevar a cabo cierta estrategia futura de inversión.

Si consideramos un movimiento Browniano $\left(W_{t}\right)_{t \in[0, T]}$ definido en un espacio fijo de probabilidad equipado con una filtración aumentada, $\left(\Omega, F,\left(F_{t}\right)_{t \in[0, T]}, P\right)$, esto es, $W_{t}$ es una variable aleatoria normal con media cero y varianza constante, $\Omega$ es un espacio muestral (el conjunto de todos los posibles resultados, en este caso los números reales), $F$ es una $\sigma$-álgebra (un conjunto de eventos relevantes, subconjuntos de números reales), $F_{t}$ representa toda la información disponible al tiempo $t \mathrm{y}$, por último, $P$ es una medida de probabilidad, entonces se supone que el valor presente de los flujos de efectivo esperados en $t, S_{t}$, es conducido por una ecuación diferencial estocástica:

$$
\mathrm{d} S_{t}=\mu\left(S_{t}, t\right) \mathrm{d} t+\sigma\left(S_{t}, t\right) \mathrm{d} W_{t}
$$


donde $\mu\left(S_{t}, t\right)$ y $\sigma\left(S_{t}, t\right)>0$ son funciones conocidas.

La valuación financiera de los proyectos energéticos que se presenta en este trabajo se realiza bajo el esquema de una opción real de expansión. En este caso, si $(1+\alpha) S_{T}-K^{\prime}$ es el valor presente neto aumentado en la proporción $\alpha$ menos el costo de la inversión adicional $K^{\prime}$ al tiempo $T$, el valor intrínseco (valor en la fecha de vencimiento) de esta opción está dado por:

$$
c_{e}\left(S_{T}, T ; \alpha, K^{\prime}\right)=\max \left((1+\alpha) S_{T}-K^{\prime}, S_{T}\right)
$$

donde $K=K^{\prime} / \alpha$. En particular, si el valor presente de los flujos de efectivo esperados es conducido por un movimiento geométrico Browniano "neutral al riesgo", es decir:

$$
\mathrm{d} S_{t}=r S_{t} \mathrm{~d} t+\sigma S_{t} \mathrm{~d} W_{t}
$$

donde $r$ es la tasa de interés libre de riesgo (de incumplimiento) y $\sigma>0$ es la volatilidad instantánea de $S_{t}$; se tiene que el valor de la opción real de expansión en $t$ está dado por:

$$
\begin{gathered}
c\left(S_{t}, t\right)=e^{-r(T-t)} \mathrm{E}\left[S_{T}+\alpha \max \left(S_{T}-K, 0 \mid F_{t}\right]\right. \\
=e^{-r(T-t)} \mathrm{E}\left[S_{T} \mid S_{t}\right]+\alpha e^{-r(T-t)} \int_{K}^{\infty}(s-K) f_{S_{T} \mid S_{t}}\left(s \mid S_{t}\right) \mathrm{d} s \\
=S_{t}+\alpha c_{\mathrm{BS}}\left(S_{t}, t\right)
\end{gathered}
$$

donde:

$$
f_{S_{T} \mid S_{t}}\left(s \mid S_{t}\right)=\left(\frac{1}{\sqrt{2 \pi(T-t) \sigma s}}\right) \exp \left\{-\frac{1}{2}\left(\frac{\ln \left(\frac{s}{S_{t}}\right)-\left(r-\frac{1}{2} \sigma^{2}\right)(T-t)}{\sigma \sqrt{T-t}}\right)^{2}\right\}
$$




$$
\begin{gathered}
\text { Valuación FinANCiERA DE PROYeCTOS DE ENERGía NUCLEAR } \\
\text { EN ARGENTINA MEDIANTE OpCIONES REALES } \\
c_{\text {BS }}\left(S_{t}, t\right)=S_{t} \Phi\left(d_{1}\right)-K e^{-r(T-t)} \Phi\left(d_{2}\right) \\
\Phi(d)=\mathrm{P}_{\varepsilon}(\varepsilon \leq d)=\int_{-\infty}^{d} \frac{1}{\sqrt{2 \pi}} e^{-\frac{1}{2} \varepsilon^{2}} \mathrm{~d} \varepsilon=1-\Phi(-d) \\
d_{1}=\frac{\ln \left(\frac{S_{t}}{K}\right)+\left(r+\frac{1}{2} \sigma^{2}\right)(T-t)}{\sigma \sqrt{T-t}} \\
d_{2}=\frac{\ln \left(\frac{S_{t}}{K}\right)+\left(r-\frac{1}{2} \sigma^{2}\right)(T-t)}{\sigma \sqrt{T-t}}=d_{1}-\sigma \sqrt{T-t}
\end{gathered}
$$

Es decir, $c_{B S}\left(S_{t}, t\right)$ es la fórmula de Black-Scholes (1973) para valuar una opción de compra. Es importante destacar que los flujos de efectivo esperados no son un activo que se compre o venda en un mercado definido, lo que genera una situación de mercados incompletos.

\section{VALUACIÓN DE PROYECTOS ENERGÉTICOS EN ARGENTINA CON OPCIONES REALES}

El liderazgo de Argentina en la operación de plantas nucleares en América Latina se explica por los 3 reactores nucleares utilizados para la generación eléctrica, denominados Atucha I, conocido como CNA I, Atucha II conocido como CNA II, y Embalse conocido como CANDU, así como siete reactores con fines de investigación (RA-0, RA-1 RA-2, RA-3 RA-4, RA-6 RA-8). Además de que se tiene acceso público de los costos reales de capital y operación de las diferentes centrales y tecnologías de generación eléctrica que justifican el uso de la información del país sudamericano para nuestra simulación con opciones reales. Los datos provienen de la Comisión Nacional de Energía Atómica de Argentina (CNEA). En 
el Cuadro 1 se presentan los costos comparativos de diferentes tipos de centrales eléctricas, donde las núcleo-eléctricas se destacan por su competitividad frente a las restantes opciones.

\section{Cuadro 1}

\section{Análisis comparativo de la estructura de costos de las centrales eléctricas en la República de Argentina}

\begin{tabular}{|c|c|c|c|c|}
\hline $\begin{array}{l}\text { Tipo de central } \\
\text { generadora }\end{array}$ & $\begin{array}{c}\text { Costo variable } \\
(\$ / M W h)^{* *}\end{array}$ & $\begin{array}{c}\text { Costo fijo } \\
(\$ / M W h)^{* * *}\end{array}$ & $\begin{array}{c}\text { Costo de capital } \\
(\$ / M W h) * *\end{array}$ & $\begin{array}{l}\text { Costo total } \\
(\$ / M W h)^{* *}\end{array}$ \\
\hline $\begin{array}{c}\text { Nuclear CNA II } \\
750 \mathrm{MW}\end{array}$ & 20.14 & 27.01 & 33.26 & 80.41 \\
\hline $\begin{array}{c}\text { Nuclear CANDU } \\
600 \mathrm{MW}\end{array}$ & 16.26 & 27.01 & 81.97 & 125.23 \\
\hline $\begin{array}{c}\text { Térmica cc } \\
800 \text { MW * } \\
\text { (3 USD/ MBTU) }\end{array}$ & 64.39 & 11.56 & 29.07 & 105.02 \\
\hline $\begin{array}{c}\text { Térmica cc } \\
800 \text { MW * } \\
\text { (4 USD } / \text { MBTU) }\end{array}$ & 86.26 & 11.56 & 29.07 & 126.89 \\
\hline $\begin{array}{c}\text { Térmica cc } \\
800 \text { MW * } \\
(5 \text { USD } / \text { MBTU) }\end{array}$ & 107.52 & 11.56 & 29.07 & 148.15 \\
\hline $\begin{array}{l}\text { Hidroeléctrica } \\
\text { Corpus Christi } \\
4,600 \mathrm{MW}\end{array}$ & 0.00 & 5.78 & 153.45 & 159.23 \\
\hline $\begin{array}{l}\text { Eólica } \\
50 \mathrm{MW}\end{array}$ & 0.00 & 9.63 & 209.32 & 218.96 \\
\hline $\begin{array}{c}\text { Solar } \\
50 \mathrm{MW}\end{array}$ & 0.00 & 15.45 & 690.74 & 706.18 \\
\hline
\end{tabular}

* Térmica $\mathrm{cc}=$ Generación de energía mediante ciclo combinado. $* *$ Costos en pesos mexicanos. Notas:

- Para esta tabla se emplearon tres tipos de costos: variable, fijo y de capital. Se entiende por costo variable al combustible, mantenimiento y suministros varios. Por costo fijo se entiende masa salarial, seguros y servicios de seguridad. Y por costo de capital se entiende la amortización del capital necesario para construir la central durante el período de vida útil de la misma.

- Cabe destacar que el costo de capital considerado para CNA-II corresponde al requerido para finalizar las obras de la misma, las cuales se encuentran en un $80 \%$ de avance desde 1987 , año en que fue paralizada por decisión política de la Administración Alfonsín.

- Nótese que en el costo variable de las centrales de ciclo combinado se han considerado 3 escenarios posibles en relación al precio del gas natural: \$3 USD el millón de BTU, \$4 USD el millón de BTU y \$5 USD el millón de BTU.

- Se consideró una tasa de amortización anual del 10\%; la vida útil de las centrales se ha tomado en 40 años para las nucleares (aunque en la actualidad la vida útil se puede extender hasta 10 años más), 20 años para las de ciclo combinado (abastecidas con gas natural), 50 años la hidroeléctrica y 10 años para las de energía solar y eólica.

Fuente: Adaptado de Fernández Franzini y De Dicco, 2007. 


\section{VALUACIÓN FINANCIERA DE PROYECTOS DE ENERGÍA NUCLEAR \\ en Argentina mediante opciones REALES}

En cuanto al desarrollo de la valuación y factibilidad de las diferentes opciones energéticas, se requiere el esquema tarifario (costo $\mathrm{MW} / \mathrm{h}$ ) del sector eléctrico argentino para realizar la valuación de forma objetiva, tomando en consideración el precio de venta del servicio en cuestión (véase el Cuadro 2).

\section{Cuadro 2}

\section{Costos tarifarios de energía eléctrica en la República de Argentina}

\begin{tabular}{|c|c|c|c|}
\hline Rubros & EDESUR & EDENOR & $\overline{E D E L A P}$ \\
\hline \multicolumn{4}{|c|}{ Tarifa $1-$ R1 } \\
\hline Cargo fijo (\$/bim) & 14785.2 & 14851.8 & 14851.8 \\
\hline Cargo variable (MWh) & 273.06 & 269.73 & 269.73 \\
\hline \multicolumn{4}{|c|}{ Tarifa 1 - R2 } \\
\hline Cargo fijo (\$/bim) & 53946 & 54245.7 & 54212.4 \\
\hline Cargo variable (MWh) & 143.19 & 139.86 & 139.86 \\
\hline \multicolumn{4}{|c|}{ Tarifa 1 - G1 } \\
\hline Cargo fijo (\$/bim) & 38295 & 38128.5 & 34398.9 \\
\hline Cargo variable (MWh) & 629.37 & 622.71 & 569.43 \\
\hline \multicolumn{4}{|c|}{ Tarifa 1 - G2 } \\
\hline Cargo fijo (\$/bim) & 286446.6 & 285114.6 & 256609.8 \\
\hline Cargo variable (MWh) & 472.86 & 469.53 & 426.24 \\
\hline \multicolumn{4}{|c|}{ Tarifa 1 - G3 } \\
\hline Cargo fijo (\$/bim) & 784881 & 780818.4 & 701497.8 \\
\hline Cargo variable (MWh) & 349.65 & 342.99 & 313.02 \\
\hline \multicolumn{4}{|c|}{ Tarifa 1 - AP } \\
\hline Cargo variable (MWh) & 293.04 & 289.71 & 289.71 \\
\hline
\end{tabular}

Tarifa: $\mathrm{R}=$ residencial, $\mathrm{G}=$ general y $\mathrm{AP}=$ alumbrado público

Notas:

- El Ente Nacional Regulador de la Electricidad (ENRE) es un organismo autárquico encargado de regular la actividad eléctrica y de controlar que las empresas del sector (generadoras, transportistas y distribuidoras EDENOR, EDESUR y EDELAP) cumplan con las obligaciones establecidas en el Marco Regulatorio y en los Contratos de Concesión.

- EDENOR = Empresa Distribuidora y Comercializadora Norte, EDESUR = Empresa Distribuidora y Comercializadora del Sur, EDELAP = Empresa Distribuidora y Comercializadora de la Plata.

- Los precios se encuentran en pesos mexicanos.

- Existen otro tipo de tarifas, pero solamente se consideró la tarifa 1, por ser ésta la más representativa.

Fuente: Adaptado de cuadro tarifario para el periodo junio 2007, <http://www.enre.gov.ar/>. 
Antes de realizar la simulación se deben hacer ciertas consideraciones. Primero, se supondrá que las fuentes generadoras de energía solamente pueden producir durante todo el año el equivalente a su capacidad nominal. Segundo, los costos reportados en el Cuadro 1, corresponden al año 0, por cada MW producido (considerando que la producción total anual es equivalente a la capacidad nominal de las fuentes de generación). Tercero, el costo de venta de la energía eléctrica será el promedio del valor entre cada uno de los operadores y entre cada una de las tarifas mostradas en el Cuadro 3 ( $\$ 350.21$ pesos por cada MW). Cuarto, la tasa libre de riesgo para el proyecto será del 10\%, y la volatilidad de los flujos de efectivo, para efectos prácticos, se fijó en $20 \%$. Quinto, el costo de mantenimiento y operación es la suma algebraica entre los costos fijos y los variables. Sexto, el valor de la inversión se considera como el costo de capital. Séptimo, el horizonte de proyección será de 10 años (tiempo en el cual se considera que la infraestructura utilizada, para la generación eléctrica mediante energía solar y eólica, llegue al final de su vida útil). Una vez realizadas las consideraciones pertinentes, los valores que se utilizarán para el desarrollo del cálculo son los que se incluyen en el Cuadro 3.

Para el desarrollo del cálculo de cada una de las opciones de inversión se utilizará la fórmula de Black-Scholes (1973). Para este caso en particular utilizaremos el call de la opción.

$$
\text { Call }=S \Phi\left(\frac{\ln (S / K)+\left(r+\sigma^{2} / 2\right) T}{\sigma \sqrt{T}}\right)-K e^{-r t} \Phi\left(\frac{\ln (S / K)+\left(r-\sigma^{2} / 2\right) T}{\sigma \sqrt{T}}\right)
$$

donde:

$S_{t} \quad=$ Valor presente de los flujos de efectivo futuros (capacidad nominal x precio de venta)

$K=$ Costos de operación (costo de operación y mantenimiento)

$r \quad=$ Tasa libre de riesgo $(10 \%)$

$T \quad=$ Tiempo de expiración (10 años)

$\sigma \quad=$ Volatilidad $(\%, 20 \%)$

$\Phi=$ Distribución acumulada normal estándar

$I_{0} \quad=$ Inversión inicial (costo de capital) 
VALUACIÓN FINANCIERA DE PROYECTOS DE ENERGÍA NUCLEAR

en Argentina mediante opCiones REALES

\section{CuAdro 3}

Estructura de costos de centrales eléctricas y flujos de efectivo esperado

\begin{tabular}{|c|c|c|c|c|}
\hline $\begin{array}{l}\text { Tipo de central } \\
\text { generadora }\end{array}$ & $\begin{array}{c}* \text { Precio de venta } \\
\quad(\$ / M W)\end{array}$ & $\begin{array}{c}* \text { *ostos de } \\
\text { operación } \\
\text { (capacidad } \\
\text { nominal } x \text { costos } \\
\text { de operación) }\end{array}$ & $\begin{array}{c}\text { *Inversión inicial } \\
\text { (capacidad } \\
\text { nominal } x \text { costos } \\
\text { de capital }\end{array}$ & $\begin{array}{c}\text { *Flujos de } \\
\text { efectivo esperados } \\
\text { (\$, Capacidad } \\
\text { nominal x precio } \\
\text { de venta) }\end{array}$ \\
\hline $\begin{array}{c}\text { Nuclear CNA II } \\
750 \mathrm{MW}\end{array}$ & 350.21 & $35,362.50$ & $24,945.00$ & $262,657.50$ \\
\hline $\begin{array}{l}\text { Nuclear } \\
\text { CANDU } \\
600 \mathrm{MW}\end{array}$ & 350.21 & $25,962.00$ & $49,182.00$ & $210,126.00$ \\
\hline $\begin{array}{c}\text { Térmica cc } \\
\text { 800 MW } \\
\text { (3 USD/ MBTU) }\end{array}$ & 350.21 & $60,760.00$ & $23,256.00$ & $280,168.00$ \\
\hline $\begin{array}{c}\text { Térmica cc } \\
800 \text { MW } \\
\text { (4 USD/ MBTU) }\end{array}$ & 350.21 & $78,256.00$ & $23,256.00$ & $280,168.00$ \\
\hline $\begin{array}{c}\text { Térmica cc } \\
800 \text { MW } \\
\text { (5 USD/ MBTU) }\end{array}$ & 350.21 & $95,264.00$ & $23,256.00$ & $280,168.00$ \\
\hline $\begin{array}{c}\text { Hidroeléctrica } \\
\text { Corpus Christi } \\
4,600 \mathrm{MW}\end{array}$ & 350.21 & $26,588.00$ & $705,870.00$ & $1,610,966.00$ \\
\hline $\begin{array}{l}\text { Eólica } \\
50 \mathrm{MW}\end{array}$ & 350.21 & 481.50 & $10,466.00$ & $17,510.50$ \\
\hline $\begin{array}{c}\text { Solar } \\
\text { 50MW }\end{array}$ & 350.21 & 772.50 & $34,537.00$ & $17,510.50$ \\
\hline
\end{tabular}

Nota: * En pesos.

Fuente: Elaboración propia.

Una vez determinados los valores y los parámetros los resultados se presentan en el Cuadro 4. En dicho cuadro se observa cómo evoluciona el valor de la opción $c(0, T)$ a través del tiempo determinado $T$, en un horizonte de 10 años. Asimismo se observa el valor de las ganancias netas obtenidas en dichos periodos de tiempo $I_{0}-c(0, T)$. Es importante aclarar que, aunque los cálculos reportados permiten inferir sobre los posibles beneficios financieros de implementar una u otra opción, las fuentes producen una cantidad distinta de Energía. En consecuencia, para efectuar una comparación efectiva sobre cada una de las alternativas se deben homogenizar los resultados, es decir, se debe escalar la producción de energía de manera uniforme. Con el objeto de uniformizar los resultados, cada una de las fuentes se escalaron a una producción de $750 \mathrm{MW}$ por año, dando como resultado la Gráfica 1. 
Francisco Álvarez Echeverría/Francisco Venegas-Martínez/Pablo López Sarabia

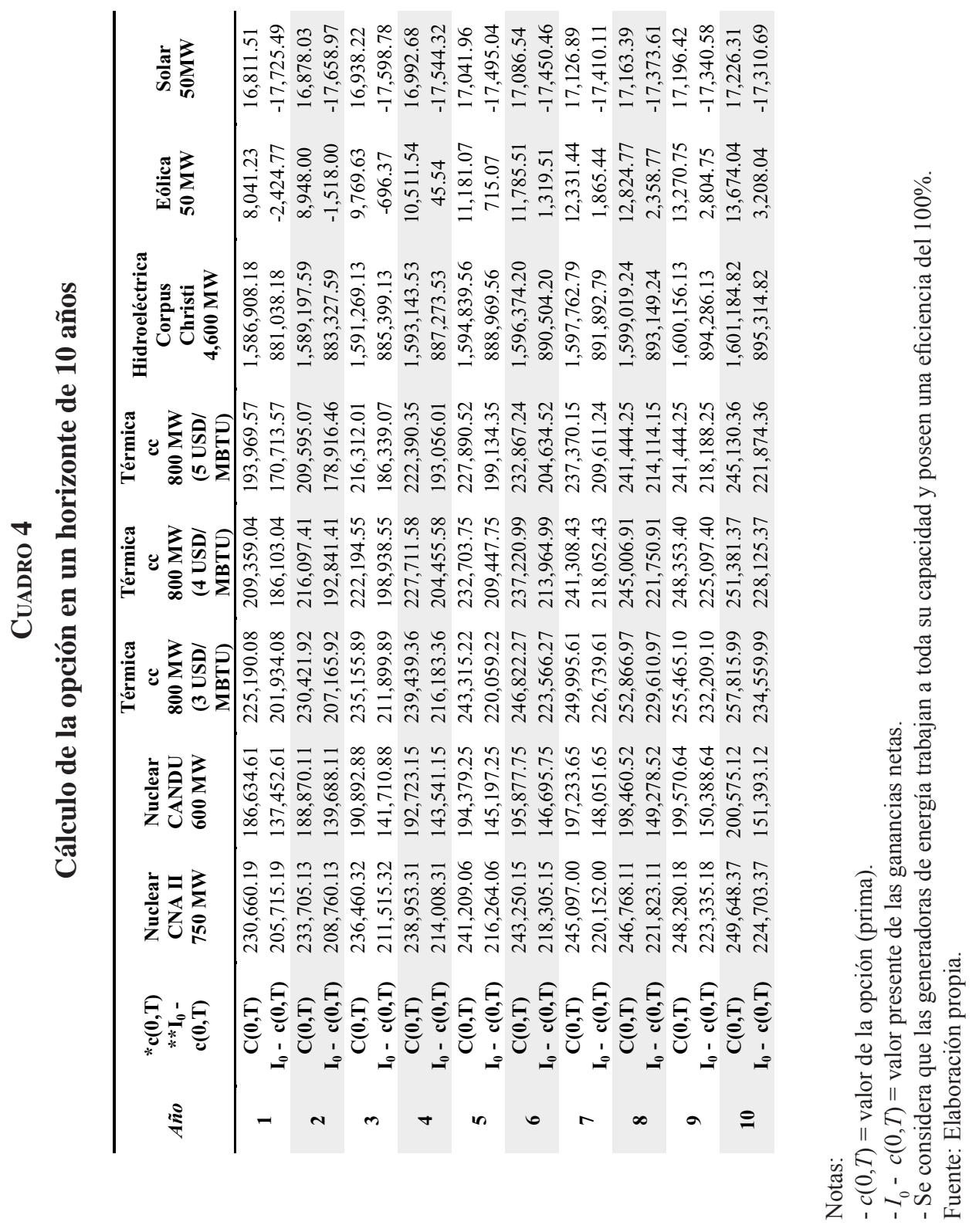


VALUACIÓN FINANCIERA DE PROYECTOS DE ENERGÍA NUCLEAR

en Argentina mediante opciones Reales

\section{GrÁFICA 1}

\section{Rendimiento de cada una de las tecnologías energéticas en Argentina valuadas financieramente con opciones reales}

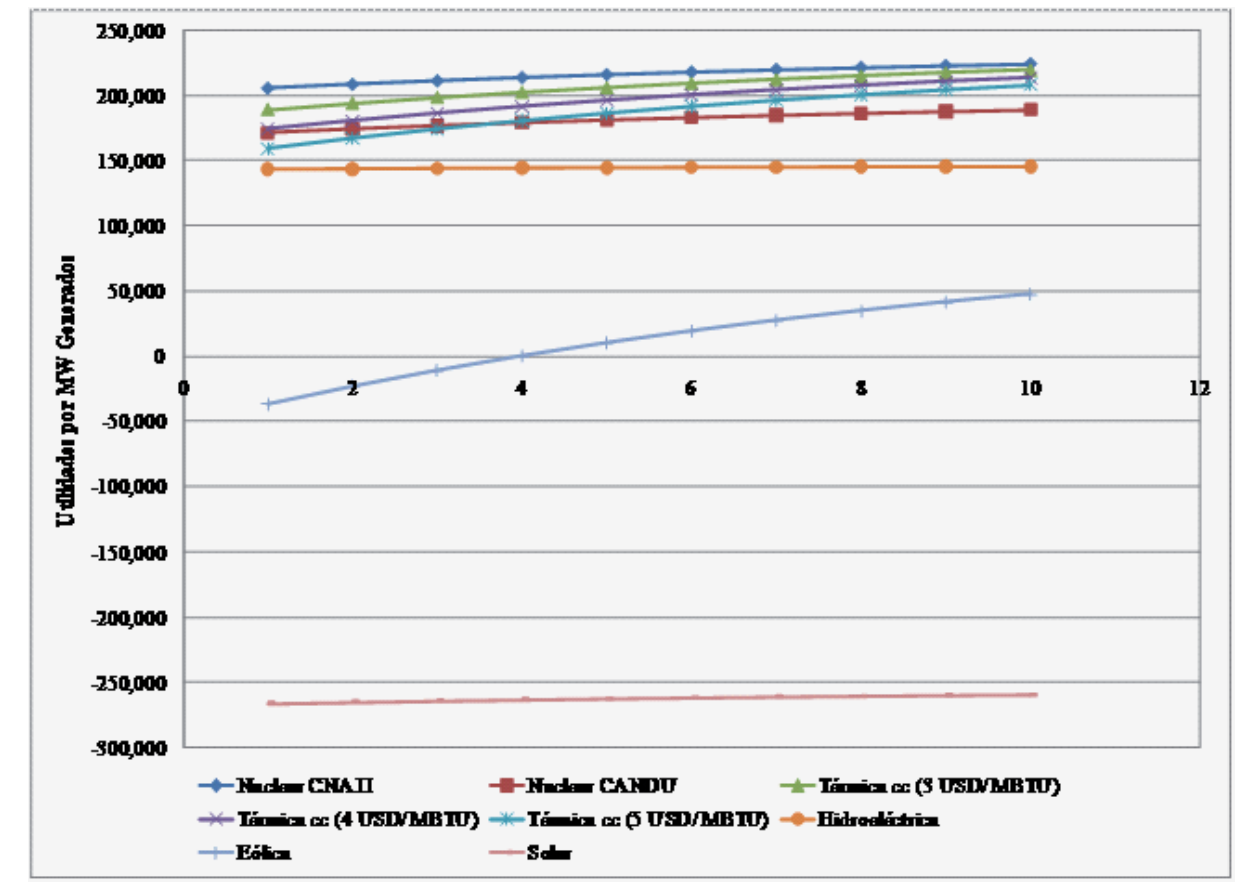

Fuente: Elaboración propia.

En la Gráfica 1 se observa que, una vez homogenizada la producción de energía de cada una de las fuentes, las centrales núcleo-eléctricas muestran un mejor desempeño (en el aspecto financiero), ya que la utilidad neta esperada proveniente del reactor nuclear CNA II supera el desempeño de las demás opciones. Con respecto a CANDU, el comportamiento a través del tiempo es estable y no tiene una variabilidad importante, esto aunado al tiempo de operación esperada del reactor. Las Termoeléctricas poseen una capacidad similar a la núcleo-eléctrica, pero existe 
el inconveniente de que el precio del gas requerido para la operación tenga una variabilidad importante en los mercados internacionales debido a la volatilidad de los precios de los combustibles fósiles. En cuanto a la hidroeléctrica involucrada en el estudio, si bien es cierto que los costos de operación y mantenimiento son bajos, la inversión requerida para este tipo de infraestructura resulta ser importante, aunado a la extensión de área requerida para su construcción, a lo anterior se agrega que su vida útil se extiende a 50 años y, asimismo se considera que, la vida estimada de una núcleo- eléctrica es de 60 años (vida estimada para la nueva generación de reactores nucleares). Dicha opción se encuentra en desventaja, tanto en desempeño como en la proyección a largo plazo como fuente generadora de energía viable. De la misma forma, se observa que la energía eólica presenta una rentabilidad marginal a partir del cuarto año, asimismo, en el caso de la energía solar, los costos asociados a estos proyectos en la actualidad resultan ser cuantiosos, lo que da origen a que la inversión no se recupere a lo largo de la vida útil del proyecto.

\section{CONCLUSIONES}

La generación de energía a través de combustibles fósiles cada vez es más riesgosa, al tratarse de fuentes no renovables y generadoras de externalidades negativas como la contaminación. Además, la volatilidad que experimentan los precios de los energéticos en el entorno mundial hace que los proyectos de inversión en esa área sean altamente riesgosos en el corto y largo plazo.

Las opciones reales son una herramienta que mejora el proceso de toma de decisiones concernientes a la inversión en activos reales, incluidos los proyectos energéticos, debido a que incorpora flexibilidad al proyecto y lo administra activamente al utilizar probabilidades de riesgo en la inversión. A pesar del potencial de las energías renovables y limpias, el costo de las mismas sigue siendo restrictivo en comparación con las que utilizan combustibles fósiles, en cuyo caso la energía nuclear es una opción confiable y factible desde el punto de vista económico y ambiental. 
VALUACIÓN FINANCIERA DE PROYECTOS DE ENERGÍA NUCLEAR

en Argentina mediante opciones Reales

\section{BIBLIOGRAFÍA}

Bazán-Perkins S. D. (2005), "La energía nuclear, una alternativa de sustentabilidad para resolver la demanda eléctrica de México (Primera parte), Ingeniería, Investigación y Tecnología, Vol. 4, No. 3, pp. 187-205.

Black, F. y M. Scholes (1973), "The Pricing of Options and Corporate Liabilities”, Journal of Political Economy, Vol. 81, No. 3, pp. 637-654.

Brealey y Myers (1991), Fundamentals Of Corporate Finance, 3era. Edición. Editorial Mcgraw-Hill.

Doraszelski, U. (2001), "The Net Present Value method versus the option value of waiting: a note on Farzin", Huisman and Kort (1998), Journal of Economic Dynamics \& Control, Vol. 25, No. 8, pp. 1109-1115.

Organismo Nacional Regulador de la Energía Eléctrica (2007), <http://www.enre. gov.ar/>.

Fernández, F. A. y R. De Dicco (2007), "Principales Características del Parque Nucleoeléctrico de Argentina”, IDICSO, Instituto de Investigación en Ciencias Sociales, Área de Recursos Energéticos y Planificación para el Desarrollo, Universidad del Salvador, <http://www.estrucplan.com.ar/articulos/ verarticulo.asp?IDArticulo $=1683>$.

Foro Nuclear (2010), <http://www.foronuclear.org/energia_nuclear_mundo.jsp>, consultado en septiembre 2010.

Lander D. M. y G. Pinches (1998), "Challenges to the practical implementation of modeling and valuing real options", The Quarterly Review of Economics and Finance, Vol. 38, Special Issue, pp. 537-567.

López Álvarez, R. (2006), “Análisis prospectivos de centrales nucleares de IV generación basadas en ciclos de Brayton indirectos", Universidad Pontificia Comillas, Escuela Técnica Superior de Ingeniería (ICAI), Madrid.

MacDougall, S. L. y R. H. Pike (2003), "Consider your Options: Changes to Strategic Value During Implementation of Advanced Manufacturing Technology", International Journal of Management Science, Vol. 31, No.1, pp. 1-15.

Pennings, E. y O. Lint (1997), “The Option Value of Advanced R\&D”, European Journal of Operational Research, 103(November), pp. 83-94. 
Francisco Álvarez Echeverría/Francisco Venegas-Martínez/Pablo López Sarabia

Venegas-Martínez, F. (2006), Riesgos financieros y económicos: Productos derivados y decisiones económicas bajo incertidumbre, México, Internacional Thomson Editores, Thomson Learning. 